Pacific Journal of Mathematics

Detrakant Extensors of Man 


\title{
EQUIVARIANT EXTENSIONS OF MAPS
}

\author{
JAN W. JAWOROWSKI
}

This paper treats extension and retraction properties in the category $\mathscr{A}_{p}$ of compact metric spaces with periodic maps of a prime period $p$; the subspaces and maps in $\mathscr{A}_{p}$ are called equivariant subspaces and maps, respectively. The motivation of the paper is the following question: Let $E$ be a Euclidean space and $a: E \times E \rightarrow E \times E$ be the involution $(x, y) \rightarrow(y, x)$, i.e., the symmetry with respect to the diagonal. Suppose that $Z$ is a symmetric (i.e., equivariant) closed subset of $E \times E$ which is an absolute retract; that is, $Z$ is a retract of $E \times E$. When does there exist a symmetric (i.e., equivariant) retraction $E \times E \rightarrow Z$ ?

This is an extension problem in the category $\mathscr{A}_{p}$. If $X$ and $Y$ are spaces in $\mathscr{A}_{p}, A$ is a closed equivariant subspace of $X$ and $f: A \rightarrow Y$ is an equivariant map, then the existence of an extension of $f$ does not, in general, imply the existence of an equivariant extension. It is shown, however, that if $A$ contains all the fixed points of the periodic map and $\operatorname{dim}(X-A)<\infty$, then a condition for the existence of an extension is also sufficient for the existence of an equivariant extension. In particular, it follows that a finite dimensional space $X$ in $\mathscr{A}_{p}$ is an equivariant ANR (i.e., an absolute neighborhood retract in the category $\mathscr{A}_{p}$ ) if and only if it is an ANR and the fixed point set of the periodic map on $X$ is an ANR. Generally speaking, the paper deals with the question of symmetry in extension and retraction problems.

1. Preliminaries. Suppose that a group $G$ acts on spaces $X$ and $Y$ and that $A$ is an equivariant subspace of $X$ (i.e., $A$ is stable under the action of $G$ ). One can then ask for conditions for the extistence of an equivariant extension of $f$; or for conditions under which the existence of an extension of $f$ implies also the existence of an equivariant extension. A general theorem of this type is due to A. Gleason [6] and R. S. Palais [12, p. 19]:

Tietze-Gleason Theorem. Let $G$ be an orthogonal group acting on a Euclidean space $E$ by means of orthogonal transformations and let $G$ act on a normal space $X$. Let $A$ be a closed equivariant subset of $X$ and let $f: A \rightarrow E$ be an equivariant map. Then there is an equivariant extension $g: X \rightarrow E$ of $f$.

This theorem is proved by first extending the map $f$ to some map $\bar{f}: X \rightarrow E$ which may not necessarily be equivariant: and then by averaging $\bar{f}$, using a Haar measure on $G$, to make it equivariant. 
Two facts play a crucial role in this proof: one is that $E$ is convex; and the other is that the action of $G$ is linear. While the second condition is not necessarily restrictive (in view of results due to Mostow [11]; Copeland and de Groot [2]; Kister and Mann [8]; the action of $G$ can be linearized), the first condition makes it impossible to apply a theorem of this type to our original problem (these two conditions are, in fact, related: by linearization of the map, the convexity of the space may be distorted).

In this paper we consider actions of $Z_{p}$, the cyclic group of a prime order $p$. In order words, we consider the category $\mathscr{A}_{p}$ whose objects are periodic homeomorphisms $a: X \rightarrow X$ of a prime period $p$ on a space $X$; i.e., $a^{p}=1$. An object $a: X \rightarrow X$ in $\mathscr{A}_{p}$ will also be denoted by $(X, \alpha)$, or simply by $X$, if the periodic map $a$ is known. A morphism in $\mathscr{A}_{p}$ from $(X, a)$ to $(Y, b)$ is a map $f: X \rightarrow Y$ consistent with the periodic maps $a$ and $b$; it will be called an equivariant map. A subspace $A$ of $X$ is said to be equivariant if it is stable under $a$, i.e., if $a A \subset A$. If $A$ is an equivariant subspace of $X$ then the periodic map $A \rightarrow A$ defined by the restriction of $a: X \rightarrow X$ of $A$ will sometimes be denoted by $a_{A}: A \rightarrow A$.

The set of the fixed points of a map $a: X \rightarrow X$ will be denoted by $F(a)$. If $a: X \rightarrow X$ is a periodic map of a prime period $p$ then $F(a)=F\left(a^{q}\right)$, for evey $q=1, \cdots p-1$.

An example of a theorem which carries over to the category $\mathscr{A}_{p}$ in a way similar to that of the Tietze theorem is the Dugundji extension theorem. In the category $\mathscr{A}_{p}$ it can be stated as follows:

Dugundu Equivariant Extension Theorem (in the category $\mathscr{A}_{p}$ ). Let $(X, a)$ be a space in $\mathscr{A}_{p}$ such that $X$ is metrizable and let $A$ be an equivariant closed subspace of $X$. Let $L$ be a locally covex vector space with a linear periodic map $b: L \rightarrow L$ of period $p$ and let $Q$ be an equivariant convex subspace of $L$. Let $f: A \rightarrow Q$ be an equivariant map. Then $f$ can be extended to an equivariant map $g: X \rightarrow Q$.

The proof is the same as that of the Tietze-Gleason theorem. By the Dugundji extension theorem there exists an extension $\bar{f}: X \rightarrow$ $Q$. We define an equivariant extension $g$ by

$$
g=\frac{1}{p} \sum_{i=1}^{p} b^{p-i} \circ \bar{f} \circ a^{i}
$$

In other words, this theorem says that $\left(L, b_{L}\right)$ is an "absolute extensor" in this category of spaces. One can likewise introduce the definitions of "absolute neighborhood extensor", "absolute retract" and "absolute neighborhood retract" in the category $\mathscr{A}_{p}$ or in other similar categories. 
Returning to our original problem of the existence of an equivariant retraction, let us now state it as follows (in the case of compact metric spaces):

Question $I$. Let $Q$ be a Hilbert cube and let $a: Q \rightarrow Q$ be a periodic map of a prime period $p$ such that $a$ is linear with respect to the linear structure on $Q$. Let $Z \subset Q$ be an equivariant closed subspace of $Q$ which is a retract of $Q$. When does there exist an equivariant retraction $Q \rightarrow Z$ ?

First, it is known that if $X$ is any separable metric space with a peiodic map $a: X \rightarrow X$ of period $p$ then there exists an equivariant embedding of $X$ in a Hilbert cube with a linear, even a distance preserving, map of period $p$. Such an embedding is known as a linearization of $(X, a)$ (see [2], Theorem II). We choose any embedding $X \subset Q$ and then define an equivariant embedding $X \rightarrow Q^{p}$ by

$$
x \mapsto\left(x, a x, \cdots, a^{p-1} x\right) .
$$

Thus the periodic map becomes a cyclic permutation of the coordinates of $Q^{p}$ (in fact, our original case was of the involution $E \times E \rightarrow E \times E$ of this form). Similarly, if $\operatorname{dim} X<\infty$, then $X$ can be equivariantly embedded in a finite-dimensional cube $I^{n}$ with an isometric periodic map.

Returning to Question $I$, let us assume therefore that there exists an equivariant retraction $r: Q \rightarrow Z$. Consider the fixed point sets $F(a)$ and $F\left(a_{Z}\right)$ of the map $a$ on $Q$ and $Z$, respectively. Then $r$ defines a retraction of $F(a)$ to $F\left(a_{z}\right)$. But since $a$ is linear, $F(a)$ is a compact convex subset of $Q$ and hence an absolute retract (it is, in fact, homeomorphic to $Q$ or to a finite-dimensional cube: see [7] and [9]). Therefore the fixed point set $F\left(a_{Z}\right)$ of $a$ would have to be an absolute retract; and this need not necessarily be the case, since there is the following example due to E. E. Floyd [4].

Floyd's example. There exists a 5-dimensional compact contractible polyhedron $Z$ with an involution $a: Z \rightarrow Z$ whose fixed point set $F\left(a_{Z}\right)$ is not contractible; in fact, $H_{1}\left(F\left(a_{Z}\right)\right) \neq 0$.

Similarily, one can construct an example of a compact AR $Z$ with an involution $a: Z \rightarrow Z$ such that $F\left(a_{Z}\right)$ is not an ANR.

Thus, in Question I, the condition that both $Z$ and $F\left(a_{Z}\right)$ be AR's is necessary for $Z$ to be an equivariant retract of $Q$; similarly, the condition that $Z$ and $F_{a}(Z)$ be ANR's is necessary for $Z$ to be an equivariant neighborhood retract of $Q$. Consequently, the question arises as to whether these conditions are also sufficient. 
Let us specify our questions as follows:

Questions. Let $Q$ be a Hilbert cube with a linear periodic map $a: Q \rightarrow Q$ of period $p$ and let $Z$ be an equivariant closed subspace of $Q$.

Question $I^{\prime}$. Suppose that both $Z$ and the fixed point set $F\left(a_{Z}\right)$ of $a$ are AR's. Is $Z$ an equivariant retract of $(Q, a)$ ?

Question $I^{\prime \prime}$. Suppose that both $Z$ and $F\left(a_{Z}\right)$ are ANR's. Is $Z$ an equivariant neighborhood retract of an equivariant neighborhood of $Z$ in $Q$ ?

The main result of this paper is to show that if $p$ is prime and the dimension of $Z$ in finite then the answer to Questions $I^{\prime}$ and $I^{\prime \prime}$ is affirmative. In fact, the following theorem will be proved:

THEOREM 1.1. Let $X$ be a compact metric space with a periodic map $a: X \rightarrow X$ of period $p$ and let $A$ be an equivariant closed subspace of $X$ containing all the fixed points, of $a$ and such that $\operatorname{dim}(X-A)<$ $\infty$. Let $Y$ be a compact metric space with a periodic map $b: Y \rightarrow Y$ of period $p$ and let $f: A \rightarrow Y$ be an equivariant map. Then:

(i) If $Y$ is an $A R$, then there exists an equivariant extension $g: X \rightarrow Y$ of $f$ over $X$;

(ii) If $Y$ is an $A N R$, then there exists an equivariant extension $g: U \rightarrow Y$ of $f$ over an equivariant neighborhood $U$ of $A$ in $X$.

We can now use Theorem (1.1) to answer our questions in the finitedimensional case; in fact, we use part (i) to answer Question $I^{\prime}$ and part (ii) to answer Question $I^{\prime \prime}$. Let us consider, for instance, the case (i) and $I^{\prime}$. Since $\operatorname{dim} Z<\infty$, there is an equivariant embedding of $\left(Z, a_{z}\right)$ in an $n$-cube $I^{n}$ : that is, an equivariant homeomorphism of $Z$ onto an equivariant subspace $Z^{\prime}$ of $I^{n}$ with a periodic map $b: I^{n} \rightarrow I^{n}$ (which can even be assumed isometric). Let us apply Theorem (1.1) to $X=I^{n}$, $A=Z^{\prime} \cup F(b)=Y$ and $f=1_{A}$ (the identity map $A \rightarrow A$ ). By Theorem (1.1), there exists an equivariant retraction $r: I^{n} \rightarrow A$. Since $F\left(b_{Z^{\prime}}\right)$ is an $\mathrm{AR}$, there exists a retraction $q: F(b) \rightarrow F\left(b_{Z^{\prime}}\right)$. The retraction $q$ defines a retraction $q^{\prime}: A \rightarrow Z^{\prime}$ by extending via the identity $Z^{\prime} \rightarrow Z^{\prime}$. The composition $q^{\prime} \circ r$ is an equivariant retraction of $I^{n}$ to $Z^{\prime}$. Now, since $I^{n}$ can be embedded as an equivariant retract of $Q$, it follows that $Z$ is an equivariant retract of $Q$.

Similarly, part (ii) of Theorem (1.1) yields an affirmative answer to Question I".

The proof Theorem (1.1) uses the classical method of replacing $X-A$ by the nerve of a covering adjusted to the equivariant category. 
It works, however, only under the assumption $\operatorname{dim}(X-A)<\infty$. It is an open question whether this finite-dimensional assumption in Theorem (1.1) is essential.

The main results of this paper have been announced in [6].

2. Linearization. We summarize some results on linearization of periodic maps (see [2]). Given a space $Z$, we denoted by $c(p, Z$ ) the periodic map of the $p$-fold Cartesian product $Z^{p}$ defined by $\left(z_{1}, \cdots, z_{p}\right) \rightarrow\left(z_{p}, z_{1}, \cdots, z_{p-1}\right)$ i.e., $c(p, Z)$ is a cyclic permutation of the coordinates.

(2.1). If $Z$ is a vector space then $\left(Z^{p}, c(p, Z)\right)$ is a vector space with the periodic map $c(p, Z)$ being linear with respect to the product vector space structure.

(2.2). If $Z$ is a metric space then $c(p, Z)$ is isometric (i.e., distance preserving) with respect to the product metric in $Z^{p}$.

(2.3). If $(X, a)$ is an object in $\mathscr{A}_{p}$ and there is an embedding $h: X \rightarrow Z$ of $X$ in a space $Z$, then there is an equivariant embedding of $(X, a)$ in $\left(Z^{p}, c(p, Z)\right)$ defined by $x \mapsto\left(h x, h(a x), \cdots, h\left(a^{p-1} x\right)\right)$.

In particular

(2.4). If $(X, a)$ is an object of $\mathscr{A}_{p}$ such that $X$ is a compact metric space, then there is an equivariant embedding of $(X, a)$ in $(Q, c)$ where $Q$ is a Hilbert cube with an isometric map $c: Q \rightarrow Q$ of period $p$. If $\operatorname{dim} X<\infty$, then there is an equivariant embedding of $(X, a)$ in $\left(I^{n}, c\right)$, where $I^{n}$ is a finite-dimensional cube with an isometric periodic map $c: I^{n} \rightarrow I^{n}$ of period $p$.

Let us also note that if $(V, a)$ is a vector space with a linear periodic map $a: V \rightarrow V$ of period $p$ and $Z$ is an equivariant convex subset of $V$ then the equivariant embedding $h: x \mapsto\left(h x, h(a x), \cdots, h\left(a^{p-1} x\right)\right)$ carries $Z$ onto a convex subset of $\left(V^{p}, c(p, V)\right)$. In particular, an $n$-cube $\left(I^{n}, a\right)$ with a linear periodic map $a: I^{n} \rightarrow I^{n}$ can be equivariantly embedded as a convex subset of $(Q, c)$, where $Q$ is a Hilbert cube with a linear periodic map $c: Q \rightarrow Q$. Using the Dugundji equivariant extension theorem we obtain the following corollary:

(2.5) If $\left(I^{n}, a\right)$ is an $n$-cube with a linear periodic may $a: I^{n} \rightarrow I^{n}$ of period $p$ then $\left(I^{n}, a\right)$ can be equivariantly embedded as an equivariant retract of a Hilbert cube $(Q, c)$ with a periodic map $c: Q \rightarrow Q$.

3. Retracts and extensors in the category $\mathscr{A}_{p}$. We summarize the definitions and main properties of retracts and extensors in the 
category $\mathscr{A}_{p}$ of spaces with $Z_{p}$-actions (compare Palais [12], p. 25) which are usually called $\boldsymbol{Z}_{p}$-retracts and $\boldsymbol{Z}_{p}$-extensors. Since the prime integer $p$ and the group $\boldsymbol{Z}_{p}$ is fixed throughout the paper (except where the results are specialized to the case $p=2$ ), we shall simply call them equivariant retracts and equivariant extensors.

Definition 3.1. An object $(Y, b)$ of $\mathscr{A}_{p}$ is said to be an equivariant absolute extensor (abbreviated to EAE) if given an object $(X, a)$ of $\mathscr{A}_{p}$ such that $X$ is a metric space, given a closed equivariant subspace $A$ of $X$ and given an equivariant map $f: A \rightarrow Y$, there is an equivariant extension $g: X \rightarrow Y$ of $f$.

An object $(Y, b)$ of $\mathscr{A}_{p}$ is said to be equivariant absolute neighborhood extensor (EANR) if given $(X, a), A$ and $f$ as above, there is an equivariant extension $g: U \rightarrow Y$ of $f$ over some equivariant neighborhood $U$ of $A$ in $X$.

Definition 3.2. An object $(X, a)$ of $\mathscr{A}_{p}$ is said to be an equivaraiant absolute retract (abbreviated to ERA) if $X$ is a metric space and for any equivariant imbedding $h:(X, a) \rightarrow(Y, b)$ in an object $(Y, b)$ of $\mathscr{A}_{p}$ such that $Y$ is a metric space and $h X$ is closed in $Y$, the image $h X$ in an equivariant retract of $(Y, b)$.

An object $(X, a)$ of $\mathscr{A}_{p}$, where $X$ is a metric space, is said to be an equivariant absolute neighborhood retract (EANR) if given $h:(X, a) \rightarrow(Y, b)$ as above, the image $h X$ is an equivariant neighborhood retract of $(Y, b)$.

The following theorems are proved in the same way as in the topological category:

THEOREM 3.3. An equivariant retract of an $E A E$ is an $E A E$; an equivariant neighborhood retract of an EANE is an EANE.

THEOREM 3.4. A Hilbert cube $(Q, c)$ with a linear periodic map $c: Q \rightarrow Q$ of a prime period $p$ is an EAE.

This is, in fact, a particular case of the Dugundji extension theorem in the category $\mathscr{A}_{p}(\S 1)$.

Theorem 3.5. Let $(X, a)$ be an object of $\mathscr{A}_{p}$ such that $X$ is a compact metric space. Then the following conditions are equivalent:

(i) $(X, a)$ is an $E A E$.

(ii) $(X, a)$ is an $E A R$.

(iii) $(X, a)$ can be equivariantly embedded as an equivariant retract of $(Q, c)$, where $Q$ is a Hilbert cube with an isometric periodic map $c: Q \rightarrow Q$ of period $p$.

Similarly, the following conditions are equivalent:

(iN) $(X, a)$ is an EANE. 
(iiN) $(X, a)$ is an EANR.

(iiiN) $(N, a)$ can be equivariantly embedded as an equivariant neighborhood retract of $(Q, c)$, where $(Q, c)$ is as above.

Moreover, if $\operatorname{dim} X<\infty$, then the Hilbert cube $(Q, c)$ can be replaced by a finite-dimensional cube $I^{n}$ with an isometric involution.

Theorem (3.5) is proved by using the linearization embeddings $(\S 2)$.

COROLlARY 3.6. The following objects of $\mathscr{A}_{p}$ are equivariant absolute retracts:

(1) A Hilbert cube $(Q, c)$ with a linear periodic map $c: Q \rightarrow Q$.

(2) An n-cube $\left(I^{n}, c\right)$ with a linear periodic map $c: Q \rightarrow Q$.

4. Equivariant coverings and replacement by polyhedra. In this section we describe the classical constructions due to Kuratowski [10] and Dugundji [2] which are used in extending maps. We adjust them spaces with periodic maps, but we restrict ourselves to compact metric spaces.

The following notation will be used: diam $S$ is the diameter of a subset $S$ of a metric space $X ; B(x, \varepsilon)$ is the open ball in $X$ of center $x$ and radius $\varepsilon$; and Conv $S$ is the convex hull of a subset $S$ of a linear space $L$.

Let $\alpha$ be a collection of subsets of $X$. If $U \in \alpha$, then $S t_{\alpha} U$ is the union of the members of $\alpha$ which meet $U$. We say that Ord $\alpha \leqq$ $n$ if every collection of $n+1$ members of $\alpha$ has an empty intersection. If $X$ is an object of $\mathscr{A}_{p}$ with a periodic map $a: X \rightarrow X$, let $a \alpha=\left\{\alpha^{q} U \mid U \in \alpha, q=1, \cdots, p-1\right\}$; the collection $\alpha$ is said to be equivariant if $a \alpha=\alpha$.

Covering Lemma 4.1. Let $(X, a)$ be an object of $\mathscr{A}_{p}$ such that $X$ is a compact metric space and let $A$ be an equivariant closed subspace of $X$ containing the fixed point set $F(a)$ of $a$. Let $\alpha$ be an equivariant open cover of $X-A$. Then there exists an equivariant countable open cover $\beta$ of $X-A$ which is a refinement of $\alpha$ and satisfies the following conditions:

(i) $\lim _{U \in \beta}(\operatorname{diam} U)=0$

(ii) If $U \in \beta$ then $\mathrm{Cl} U \subset X-A$.

(iii) Every neighborhood of $A$ in $X$ contains all but a finite number of elements of $\beta$.

(iv) For every $U \in \beta$, the sets $S t_{\beta} U, a\left(S t_{\beta} U\right), \cdots, a^{p-1}\left(S t_{\beta} U\right)$ are mutually disjoint.

(v) If $\operatorname{dim}(X-A) \leqq n$ then Ord $\beta \leqq p(n+1)$. 
Proof. We can assume that $d$ is an equivariant distance function on $X$, i.e., that $a: X \rightarrow X$ is isometric. Let $A_{0}=X$,

$$
\begin{array}{rlrl}
A_{i} & =\left\{x \in X \mid d(x, A)<\frac{1}{2^{i}}\right\}, & i=1,2, \cdots \\
C_{i}=\mathrm{Cl}\left(A_{i}\right)-A_{i+1}, & i=0,1, \cdots
\end{array}
$$

The sets $C_{i}$ are compact. Since $p$ is prime, the group $Z_{p}$ acts freely on $X-A$, i.e., for every $x \in X-A$, the orbit $\left\{x, a x, \cdots, a^{p-1} x\right\}$ consists of $p$ distinct points. It follows that, for each $i=0,1, \cdots$, there is a positive number $\eta_{i}$ such that

$$
d\left(x, a^{p} x\right) \geqq \eta_{i} \quad \text { for every } q=1, \cdots, p-1 \text { and } x \in C_{i} .
$$

For each $i=0,1, \cdots$, there is a finite open cover $\gamma_{i}$ of $C_{i}$ by open balls in $X$ with centers in $C_{i}$ and radii $r_{i}>0$ such that $\gamma_{i}$ is a refinement of $\alpha$ and

$$
\begin{gathered}
r_{i} \leqq 2^{-i-3} \\
r_{i+1} \leqq \frac{1}{2} r_{i} \\
6 \gamma_{i} \leqq \eta_{i+1} .
\end{gathered}
$$

Let $\beta_{i}=\gamma_{i} \cup a\left(\gamma_{i}\right) \cup \cdots \cup a^{p-1}\left(\gamma_{i}\right)$ and $\beta=\beta_{0} \cup \beta_{1} \cup \cdots$. Then $\beta$ is an equivariant countable open cover of $X-A$ and is a refinement of $\alpha$ since $\alpha$ is equivariant. Conditions (i), (ii) and (iii) follow directly from the construction of $\beta$.

Let us verify condition (iv). Observe that by (4.3) and (4.4), and since the map $a$ is isometric, every member of $\beta$ is an open ball contained in $A_{j-1}-\mathrm{Cl}\left(A_{j+1}\right)$, for some $j=1,2, \cdots$. Thus if a member $V$ of $\beta$ meets a member $U$ of $\beta_{i}$ then $V \in \beta_{i-1} \cup \beta_{i} \cup \beta_{i+1}$ and, by (4.3) and (4.4), $U$ and $V$ are open balls of radii $\leqq r_{i-1} \leqq 2^{-i-2}$.

Since the map $a: X \rightarrow X$ is isometric, to prove condition (iv) it suffices to show that $\left(S t_{\beta} U\right) \cap\left(a^{q}\left(S t_{\beta} U\right)\right)=\varnothing$ for each $U \in \beta$ and $q=$ $1, \cdots, p-1$. Let $U \in \beta$ and suppose that $U \in \beta_{i}$. Then the center $x$ of the open ball $U$ is in $C_{i}$. By the remark above, for every $y \in$ $S t_{\beta} U$ we have $d(x, y)<r_{i}+2 r_{i-1}<3 r_{i-1}$. If we suppose that $\left(S t_{\beta} U\right) \cap$ $\left(a^{q}\left(S t_{\beta} U\right)\right) \neq \varnothing$ for some $q=1, \cdots, p-1$, then, since the map $a$ is is isometric, it would follow that $d\left(x, a^{q} x\right)<6 r_{i-1} \leqq \eta_{i}$, which contradicts to (4.5) and (4.2).

Suppose now that $\operatorname{dim}(X-A) \leqq n$. Then the open cover $\beta$ has an open refinement $\omega$ of order $\leqq n+1$. Since $C_{i}$ is compact, there is a finite subcollection $\omega_{i}$ of $\omega$ which covers $C_{i}$. Let $\beta_{i}^{\prime}=\omega_{i} \cup$ $a\left(\omega_{i}\right) \cup \cdots \cup a^{p-1}\left(\omega_{i}\right)$ and $\beta^{\prime}=\beta_{0}^{\prime} \cup \beta_{1}^{\prime} \cup \cdots$. Then $\beta^{\prime}$ is an equivariant 
countable open refinement of $\beta$ which satisfies the conditions corresponding to (i), (ii) and (iii). Condition (iv) for $\beta^{\prime}$ follows from the fact that it holds for $\beta$ and that $\beta^{\prime}$ is a refinement of $\beta$.

Let us verify condition (v). Suppose that $\sigma$ is a subcollection of $\beta^{\prime}$ containing $p$ distinct elements whose intersection is nonempty. For each $W \in \sigma$, there is an integer $q, 0 \leqq q<p$, such that $a^{q} W \in \omega$; let $q(W)$ denote the smallest integer with this property. Then this defines a map $q: \sigma \rightarrow\{0, \cdots, p-1\}$. Note that $\operatorname{Card} q^{-1}(j) \leqq n+1$, for each $j=0, \cdots, p-1$. For if $W_{0}, \cdots, W_{r}$ are distinct elements of $q^{-1}(j)$, then $a^{j}\left(W_{0}\right), \cdots, a^{j}\left(W_{r}\right) \in \omega$ and $a^{j}\left(W_{0}\right) \cap \cdots \cap a^{j}\left(W_{r}\right) \neq \varnothing$ and, consequently, $r \leqq n$, since Ord $\omega \leqq n+1$. Since $\sigma=q^{-1}(0) \cup \cdots \cup$ $q^{-1}(p-1)$, it follows that Card $\sigma \leqq p \cdot(n+1)$.

This completes the proof. Let us observe that conditions (i), (ii) and (iii) imply the following lemma.

LeMma 4.6. If $\beta$ is a cover constructed in Lemma (4.1), then for every $x \in A$ and for every neighborhood $V$ of $x$ in $X$ there exists a neighborhood $W$ of $x$ in $X$ such that if $U \in \beta$ and $U \cap W \neq \varnothing$ then $U \subset V$.

(4.7) Lemma (Replacement by polyhedra). Let $(X, a)$ be an object of $\mathscr{A}_{p}$ such that $X$ is a compact metric space and let $A$ be an equivariant closed subspace of $X$ containing the fixed point set $F(a)$ of $a$. Then there exists an object $(Z, c)$ in $\mathscr{A}_{p}$ such that $Z$ is a Hausdorff space with a periodic map $c: Z \rightarrow Z$ and:

(i) $Z$ contains $A$ as an equivariant subspace.

(ii) $Z-A$ has a countable locally finite triangulation $K,|K|=Z-A$, such that the map $c$ is simplicial and free on $K$.

(iii) There is an equivariant map of pairs: $(X, X-A) \rightarrow(Z,|K|)$ which is the identity on $A$.

(iv) There is an equivariant retraction $r^{0}: A \cup\left|K^{0}\right| \rightarrow A$.

(v) If $\operatorname{dim}(X-A) \leqq n$ then $\operatorname{dim} K \leqq p \cdot(n+1)-1$.

Proof. Let $\beta$ be an equivariant open over of $X-A$ satisfying the conditions of the Covering Lemma (4.1). Let $K$ be the nerve of $\beta$ and $Z$ be the disjoint set sum of $A$ and $|K|$. Given a member $U$ of $\beta$, we shall also be denoting by $U$ the vertex of $K$ corresponding to $U$; and $S t_{L} U$ will denote the open star of the vertex $U$ in the complex $K$, while $S t_{\beta} U$ will, as before, denote the union of the members of $\beta$ intersecting $U$.

For a subset $S$ of $X$, let $\hat{S}$ denote the union of $A \cap S$ and of the open stars of the vertices corresponding to the members of $\beta$ which are contained in $S$; i.e., $\hat{S}=(A \cap S) \cup\left(\cup\left[S t_{K} U \mid U \subset S\right]\right)$. The space $Z$ is topologized by means of the subbasis consisting of all the open 
subsets of $|K|$ and all the sets of the form $\hat{U}$, where $U$ is an open subset of $X$.

Before we proceed with the rest of the proof, we shall establish the following lemma:

Lemma 4.8. For every $x \in A$ and every neighborhood $V$ of $x$ in $X$, there is a neighborhood $O_{V}$ of $x$ in $Z$ such that if $y \in O_{V} \cap(Z-A)$ and $s$ is an open simplex of $K$ containing $y$ then all the vertices of $s$ (as members of the cover $\beta$ ) are contained in $V$.

Proof. Given a neighborhood $V$, we choose a neighborhood $W$ of $x$ according to Lemma (4.6). Let $O_{V}=\hat{W}$. Then if $y \in O_{V} \cap(Z-A)$ and $s$ is the carrier of $y$ in $K$, some vertex $U$ of $s$ is contained in $W$; and all the other vertices are contained in $V$ since they meet $U \subset W$.

Continuation of the proof of (4.7). The fact that $Z$ is Hausdorff follows readily from lemma (4.8). Since the cover $\beta$ is equivariant, it follows that $a: X \rightarrow X$ defines a periodic map on $K$ which, together with the map $a$, define a periodic map $c: Z \rightarrow Z$ of period $p$. The continuity of $c$ follows from the fact that $\beta$ is equivariant; and condition (iv) of (4.1) implies that the map $c$ is free on $K$. Thus conditions (i) and (ii) hold.

The map $\mu:(X, X-A) \rightarrow(Z, Z-A)$ of condition (iii) is defined by the identity on $A$ and a canonical map $X-A \rightarrow|K|$ of $X-A$ into the space of the nerve of the covering $\beta$ which can be described as follows: if $x \in X-A$, then the barycentric coordinates of $\mu x$ with respect to the vertex $U$ of $K$ is

$$
\frac{d(x, X-U)}{\sum_{V \in \beta} d(x, X-U)} .
$$

Since $\beta$ is equivariant and the map $a$ is isometric with respect to $d$, it follows that $\mu: X \rightarrow Z$ is equivariant. The continuity of $\mu$ follows easily from the definition of the topology, just as in [2].

A retraction $r^{0}: A \cup\left|K^{0}\right| \rightarrow A$ may be defined as follows. Let $\Lambda^{0}$ denote the set of orbits of the map $c$ on $K^{0}$ and let $\varphi: \Lambda^{0} \rightarrow K^{0}$ be any cross-section of the identification map $K^{0} \rightarrow \Lambda^{0}$. Let $N^{0}=\varphi\left(\Lambda^{0}\right)$. Since $c$ acts freely on $K^{0}$, it follows that $K^{0}$ is the disjoint union $K^{0}=N^{0} \cup c\left(N^{0}\right) \cup \cdots \cup c^{p-1}\left(N^{0}\right)$; thus for each vertex $V$ of $K^{0}$ there is a unique vertex $U$ of $N^{0}$ and a unique integer $j, 0 \leqq j<p$, such that $V=c^{j} U$. Given a vertex $U$ of $N^{0}$, let $r^{0} U$ denote any point of $A$ such that $d(U, A)=d\left(U, r^{0} U\right)$ (such a point exists since $A$ is compact). If $V$ is any vertex of $K^{0}$, choose $U \in N^{0}$ and an integer $j$ as 
above such that $V=c^{j} U$ and define $r^{0} V=a^{j}\left(r^{0} U\right)$. Since the map $a$ is isometric, we have $d(V, A)=d\left(V, r^{0} V\right)$. Defining $r^{0}$ to be the identity on $A$, we obtain a retration $r^{0}: A \cup\left|K^{0}\right| \rightarrow A$. To prove the continuity of $r^{0}$, it suffices to consider the restriction $r^{0} \mid\left(A \cup N^{0}\right)$, since the sets $A \cup\left(a^{j} N^{0}\right)$ are closed and the intersection of any two of them is $A$. Thus let $U$ be a vertex of $N^{0}$, let $z=r^{0} U$ and let $B=B(z, \varepsilon)$ be an open ball with center $z$ and radius $\varepsilon$. Let $V=B(z, \varepsilon / 3)$ and let $O_{V}$ be a corresponding neighborhood of $x$ in $Z$ satisfying the assertion of Lemma (4.8). Then $r^{0}\left(\left(A \cup N^{0}\right) \cap O_{V}\right) \subset B$. Moreover, $r^{0}$ is equivariant by its definition.

Now, if $\operatorname{dim}(X-A) \leqq n$, then by (4.1), (v), Ord $\beta \leqq p(n+1)$ and hence $\operatorname{dim} K \leqq p(n+1)-1$. This proves condition $(\mathrm{v})$.

REMARK. One can easily show that the space $Z$ is, in fact, compact and metrizable.

5. Proof of the extension Theorem (1.1). By (2.4) we can assume that $Y$ is an equivariant subspace of a Hilbert cube $Q$ with an isometric periodic map $b: Q \rightarrow Q$ of period $p$ such that the map $Y \rightarrow Y$ is the restriction of $b$.

We shall first prove case (ii) of (1.1). Suppose that $Y$ is an ANR. Then there is an equivariant compact neighborhood $C$ of $Y$ in $Q$ and a (not necessarily equivariant) retraction $r: C \rightarrow Y$. Let $\delta=d(Y, Q-C)$; then $\delta>0$. By the uniform continuity of $r$ there exists a function $\eta: R_{+} \rightarrow R_{+}$( $R_{+}=$the set of positive numbers) below the diagonal $(\eta(\varepsilon) \leqq \varepsilon)$ such that

(5.1) If $y \in C$ and $d(y, Y) \leqq \eta(\varepsilon)$ then $d(y, r y) \leqq \varepsilon$.

Consequently, $d\left(b^{j} y, b^{j} r y\right) \leqq \varepsilon$, for every $j=0, \cdots, p-1$, since $b$ is isometric.

Let $n=p \cdot(\operatorname{dim}(X-A)+1)-1$. Define a sequence of positive numbers $\varepsilon_{0}, \cdots, \varepsilon_{n}$ as follows:

$$
\varepsilon_{n}=\delta ; \quad \varepsilon_{m-1}=\frac{1}{2} \eta\left(\frac{\varepsilon_{m}}{4}\right) \text { for } 0<m \leqq n .
$$

By the uniform continuity of $f$ there is a $\hat{\xi}>0$ such that if $x, x^{\prime} \in$ $A$ and $d\left(x, x^{\prime}\right) \leqq \xi$ then $d\left(f x, f x^{\prime}\right) \leqq \varepsilon_{0}$.

Let $(Z, c)$ be a space with a periodic map $c: Z \rightarrow Z$ of period $p$, a triangulation $K$ of $Z-A$, an equivarient map $\mu: X \rightarrow Z$ and an equivariant retraction $r^{0}: A \cup\left|K^{0}\right| \rightarrow A$ as provided by the Replacement Lemma (4.7). Let $L$ be the subcomplex of $K$ consisting of the simplices $s$ of $K$ such that $d\left(r^{0} U, r^{0} V\right)<\xi$ for all vertices $U, V$ of $s$. 
Lemma 5.3. $A \cup|L|$ is an equivariant neighborhood of $A$ in $Z$.

Proof. Let $z \in A$. By the countinuity of $r^{0}$ there is a neighborhood $N$ of $z$ in $Z$ such that $d\left(r^{0} U, z\right)<\xi / 2$ for every $U \in\left(A \cup K^{0}\right) \cap$ $N$. Thus $N \subset A \cup|L|$ since every simplex of $K$ in $N$ is in $L$.

We shall construct an extension of $f$ over $A \cup|L|$. By induction, we construct a sequence of equivariant maps

$$
h_{m}: A \cup\left|L^{m}\right| \longrightarrow Y
$$

such that $h_{m}$ extends $h_{m-1}$ and that the following condition (5.4.m) holds:

$$
\operatorname{diam}\left(h_{m} s\right) \leqq \varepsilon_{m}, \quad \text { for each } m \text {-simplex } s \text { of } L^{m} \text {. }
$$

Define $h_{0}: A \cup\left|L^{0}\right| \rightarrow Y$ by $h_{0} U=f r^{0} U$, for each vertex $U$ of $L$. Suppose that $h_{m-1}: A \cup\left|L^{m-1}\right| \rightarrow Y$ is defined so that condition (5.4.m-1) holds. Let $M=L^{m}-L^{m-1}$ be the set of the $m$-simplices of $L$; and let $\Lambda$ denote the set of orbits of the simplices of $M$ under the map c. Let $\varphi: \Lambda \rightarrow M$ be any cross-section of the identification map $M \rightarrow$ $\Lambda$ and $N=\varphi(\Lambda)$. Since $c$ acts freely on $K$, it follows that $M$ is the disjoint union $M=N \cup(c N) \cup \cdots \cup\left(c^{p-1} N\right)$ and thus for each simplex $t$ of $M$ there is a unique simplex $s$ of $N$ and a unique integer $j, 0 \leqq$ $j<p$, such that $t=c^{j} s$.

By (5.4.m-1) we have for each simplex $s$ of $M$

$$
\operatorname{diam}\left(h_{m-1}(\dot{s})\right) \leqq 2 \varepsilon_{m-1}
$$

and $2 \varepsilon_{m-1} \leqq \eta\left(\varepsilon_{m} / 4\right) \leqq \varepsilon_{m} / 4 \leqq \delta$. Therefore Conv $\left(h_{m-1}(\dot{s})\right) \subset C$.

Let $t$ be a closed $m$-simplex of $M$. Choose a simplex $s$ of $N$ and an integer $j, 0 \leqq j<p$, such that $t=c^{j} s$; thus $t \in c^{j} N$. The map $h_{m-1} \mid \dot{s}: \dot{s} \rightarrow Y \subset C$, where $\dot{s}$ is the boundary of $s$, can be extended in Conv $\left(h_{m-1}(\dot{s})\right)$ to a map $u: s \rightarrow C$. Then both $u$ and $r \circ u: s \rightarrow Y$ extend $h_{m-1} \mid \dot{s}$. Note that if $x \in s$ then by (5.2),

$$
d(u x, Y) \leqq 2 \varepsilon_{m-1}=\eta\left(\frac{\varepsilon_{m}}{4}\right)
$$

and by (5.1),

$$
d(r u x, u x) \leqq \frac{\varepsilon_{m}}{4}
$$

Therefore

$$
\operatorname{diam}((r u) s) \leqq \operatorname{diam}(u s)+2 \cdot \frac{\varepsilon_{m}}{4} \leqq \frac{\varepsilon_{m}}{4}+\frac{\varepsilon_{m}}{2}<\varepsilon_{m} \cdot
$$


Let $v^{(t)}: t \rightarrow Y$ be defined by

$$
v^{(t)}=b^{j} \circ r \circ u \circ c^{p-j} .
$$

Then the map $v^{(t)}$ agrees with $h_{m-1}$ on $\dot{t}$, since $h_{m-1}$ is equivariant. It follows that the maps $v^{(t)}, t \in M$, together with $h_{m-1}$, define an equivariant map

$$
h_{m}: A \cup\left|L^{m-1}\right| \cup|M|=A \cup\left|L^{m}\right| \longrightarrow Y \text {. }
$$

The fact that $h_{m}$ satisfies the inductive condition (5.4.m) follows from the fact that $v^{(t)}, t \in M$, satisfies it by (5.5), (5.6) and since $b$ is isometric.

This completes the inductive step of the construction of $h_{m}$. The maps $h_{m}$ define a map $h: A \cup|L| \rightarrow Y$ by $h \mid(A \cup|L|)=h_{m}$. The map $h$ is continuous on $|L|$ since it is defined simplicially there. Hence, it suffices to prove the continuity of $h$ on $A$. Let $z \in A$ and let $B(h z, \varepsilon)$ be an open $\varepsilon$-ball in $Y$ with center $f z=h z$. Let $\varepsilon_{n}=(1 / 2) \varepsilon$ and let positive numbers $\varepsilon_{0}, \cdots, \varepsilon_{n-1}$ be constructed as in (5.1). By the continuity of the maps

$$
A \cup\left|K^{0}\right| \stackrel{r^{0}}{\longrightarrow} A \stackrel{f}{\longrightarrow} Y
$$

there is a neighborhood $G$ of $z$ in $Z$ such that $f(A \cap G) \subset B(f z, \varepsilon / 2)$, $G \cap|K|$ is the union of open simplices of $K$, and for each simplex $s$ of $K$ in $G, f r^{0}\left(s^{0}\right) \subset B\left(f z, \varepsilon_{0} / 2\right)$ (here $s^{0}$ denotes the set of the vertices of $s$ ). Then diam $\left(s^{0}\right)<\varepsilon_{0}$ and, by the construction of (5.1), it follows that $\operatorname{diam}(h s)<\varepsilon / 2$. Therefore $h G \subset B(h z, \varepsilon)$. This completes the proof in case (ii).

In case (i), when $Y$ is an $A R$, there is retraction $r: Q \rightarrow Y$, i.e., we may take $C=Q$, which is convex. In this case the construction simplifies: we may take $\varepsilon_{n}=\infty$ which makes conditions (5.1) and (5.2) vacuous and $L=K$. By inductions we can define a map $h: A \cup|K| \rightarrow$ $Y$ as before. The continuity of $h$ must, however, be proved as in case (ii), by using the numbers defined in (5.1).

In either case, we have constructed a symmetric map $h: A \cup$ $|L| \rightarrow Y$, where $A \cup|L|$ is a symmetric neighborhood of $A$ in $Z$ in case (ii) and $L=K$ in case (i). Define $g=h \circ \mu \mid \mu^{-1}(A \cup|L|): \mu^{-1}(A \cup|L|) \rightarrow$ $Y$. Then $h$ is a symmetric extension of $f$ over the symmetric neighborhood $\mu^{-1}(A \cup|L|)$ of $A$ in $X$ which in case (i) is the whole of $X$.

This completes the proof.

\section{Equivariant absolute retracts.}

THEOREM 6.1. Let $(X, a)$ be an object of $\mathscr{A}_{p}$ such that $X$ is a compact metric space with $\operatorname{dim} X<\infty$. Then: 
(i) $X$ is an EAR iff both $X$ and the fixed point set $F(a)$ are $A R$ 's. (ii) $X$ is an EANR iff both $X$ and the fixed point set $F(a)$ are $A N R$ 's.

Proof. By (2.4) we can assume that $(X, a)$ is equivariantly embedded in a finite-dimensional cube $I^{n}$ with an isometric periodic map $a: I^{n} \rightarrow I^{n}$ of period $p$ which we still denote by $a: I^{n} \rightarrow I^{n}$. Let $F=F\left(a_{I^{n}}\right)$; then $F\left(a_{X}\right)=F \cap X$.

We shall prove case (ii); case (i) is just simpler and was done in $\S 1$. If $X$ is an EANR then there is an equivariant retraction $r: W \rightarrow X$ of an equivariant neighborhood $W$ of $X$ in $I^{n}$ to $X$. The retraction $r$ defines a retraction of $F \cap W$ to $F \cap X$. Since $a: I^{n} \rightarrow I^{n}$ is isometric, $F$ is convex and compact, hence it is an AR (in fact, it is homeomorphic to a cube). Thus both $X$ and $F \cap X$ are ANR's.

Suppose now that both $X$ and $F \cap X$ are ANR's. Then by the Addition Theorem for ANR's ([1], p. 90), it follows that $F \cup X$ is an ANR. By the Equivariant Extension Theorem (1.1), the identity $F \cup X \rightarrow F \cup X$ can be extended to an equivariant retraction $r: U \rightarrow$ $F \cup X$, where $U$ is an equivariant neighborhood of $F \cup X$ in $I^{n}$ 。 Since $F \cap X$ is an ANR, there is a neighborhood $V$ of $F \cap X$ in $F$ and a retraction $q: V \rightarrow F \cap X$. Note that $V \cup X$ is a neighborhood of $X$ in $F \cup X$. Let $U_{0}=r^{-1}(V \cap X)$. Then $U_{0}$ is an equivariant neighborhood of $X$ in $I^{n}$ and the map $U_{0} \rightarrow X$ defined by $x \mapsto q r x$ is an equivariant retraction of $U_{0}$ to $X$.

Since the cube $I^{n}$ with an isometric periodic map period $p$ is an EAR (see (3.6)), it follows that $X$ is an EANR.

We thus have an answer to our original question.

CoRollary 6.2. Let $E$ be a Euclidean space, let $F$ be the diagonal of $E \times E$ and let $X$ be an equivariant compact subset of $E \times E$ (with respect to the involution $(x, y) \rightarrow(y, x))$. Then $X$ is an equivariant retract of $E \times E$ if and only if $X$ is a retract of $E \times E$ and $F \cap X$ is a retract of $F$.

For, in this case, $F$ is the fixed point set of the involution $E \times$ $E \rightarrow E \times E$.

Just as in Theorem (1.1), it is an open question whether the finitedimensional assumption in Theorem (6.1) is essential:

Question 6.3. Does there exist a space with an involution $a: X \times$ $X$ such that both $X$ and the fixed point set $F(a)$ are AR's but $X$ is not an EAR?

More specifically, let $Q$ be a Hilbert cube and consider the symmetry $Q \times Q \rightarrow Q \times Q$ with respect to the diagonal $F$ of $Q \times Q$. Let 
$X$ be a symmetric subset of $Q \times Q$ such that $X$ is a retract of $Q \times$ $Q$ and $F \cap X$ is a retract of $F$. Does there exist a symmetric retraction of $Q \times Q$ to $X$ ?

7. Equivariant homotopy. As an application of the previous results, we prove in this section two equivariant homotopy extension theorems.

Definition 7.1. If $(X, a)$ is an object of $\mathscr{A}_{p}$ and $I$ is the unit interval then an equivariant homotopy is an equivariant map $h: X \times$ $I \rightarrow Y$ to an object $(Y, b)$ of $\mathscr{A}_{p}$, where the periodic map on $X \times I$ is $a \times 1_{I}: X \times I \rightarrow X \times I$.

If $A$ is an equivariant subspace of $X$ then the maps $a$ and $a \times 1_{L}$ define a periodic map $\left(a \times 1_{I}\right)_{T}: T \rightarrow T$, where $T=(X \times\{0\}) \cup(A \times I) \subset$ $X \times I$; it is the restriction of $a \times 1_{I}$. The following lemma is an equivariant version of the Dowker lemma used in extending homotopies:

Lemma 7.2. Let $(X, a)$ be an object of $\mathscr{A}_{p}$ such that $X$ is a metric space, let $A$ be a closed equivariant subspace of $X$ and let $g:\left(T,\left(a \times 1_{I}\right)_{T}\right) \rightarrow(Y, b)$ be an equivariant map, where $(Y, b)$ is an object $\mathscr{A}_{p}$. If $g$ can be extended to an equivariant map $g^{\prime}: U \rightarrow Y$ of an equivariant neighborhood $U$ of $T$ in $X \times I$ then $g$ can be extended to an equivariant map $h: X \times I \rightarrow Y$.

Proof. Choose an equivariant neighborhood $V$ of $A$ in $X$ such that $(\mathrm{Cl} V) \times I \subset U$ and an equivariant Urysohn function $u: X \rightarrow I$ which is 1 on $A$ and 0 on $X-V$; for instance, $u$ may be defined by using an equivariant distance function $d$ on $X$ with the usual formula:

$$
u x=\frac{d(x, X-V)}{d(x, A)+d(x, X-V)} .
$$

Then we define $h(x, t)=g^{\prime}(x,(u x) \cdot t)$.

THeOREm 7.3. Let $(X, a)$ be an object of $\mathscr{A}_{p}$ such that $X$ is a metric space, let $(Y, b)$ be an EANE and let $f: X \rightarrow Y$ be an equivariant closed subspace of $X$. Then any equivariant homotopy of $f \mid A$ can be extended to an equivariant homotopy of $f$.

This follows from (7.2) and (3.1). Similarly, (7.2) and (1.1) yield the following result:

Theorem 7.4. Let $(X, a)$ be an object of $\mathscr{A}_{p}$ such that $X$ is a compact metric finite-dimensional space, let $A$ be a closed equivariant 
subspace of $X$ containing all the fixed points of $a$, let $(Y, b)$ be an object of $\mathscr{A}_{p}$ such that $Y$ is a compact $A N R$, and let $f: X \rightarrow Y$ be an equivariant map. Then any equivariant homotopy of $f \mid A$ can be extended to an equivariant homotopy $f$.

The author is indebted to Richard J. Allen who read the manuscript and helped to remove many mistakes.

\section{REFERENCES}

1. K. Borsuk, Theory of retracts, Monogr. Mat. 44, Warszawa 1967.

2. A. H. Copeland, Jr. and J. de Groot, Linearization of homeomorphisms, Math. Ann., 144 (1961), 80-92.

3. J. Dugundji, An extension of Tietze's theorem, Pacific J. Math., 1 (1951), 69-77.

4. E. E. Floyd, Examples of fixed point sets of periodic maps, Ann. of Math., 55 (1952), 167-171.

5. A. Gleason, Spaces with a compact Lie group of transformations, Proc. Amer. Math. Soc., 1 (1950), 35-43.

6. J. W. Jaworowski, Extensions of maps in spaces with periodic homeomorphisms, Bull. Amer. Math. Soc. (1972),

7. O.-H. Keller, Die Homoimorphie der kompalten konvexen Mengen im Hilbertschen Raum, Math. Ann., 105 (1931), 748-758.

8. J. M. Kister and L. N. Mann, Equivariant imbeddings of compact Abelian Lie groups of transformations, Math. Ann., 148 (1962), 89-93.

9. V. L. Klee, Convex bodies and periodic homeomorphisms in the Hilbert space, Trans. Amer. Math. Soc., 74 (1953), 10-43.

10. K. Kuratowski, Sur les espaces localment convexes et peaniens en dimension n, Fund. Math., 24 (1935), 269-287.

11. G. D. Mostow, Equivariant embeddings in Euclidean space, Ann. of Math., 65 (1957), 432-446.

12. R. S. Palais, The classification of G-spaces, Mem. Amer. Math. Soc., 36, Providence, R. I., 1960.

Received December 23, 1971 and in revised form March 17, 1972.

INDIANA UNIVERSITY 


\section{PACIFIC JOURNAL OF MATHEMATICS}

EDITORS

\author{
H. SAMELSON \\ Stanford University \\ Stanford, California 94305 \\ C. R. HOBBY \\ University of Washington \\ Seattle, Washington 98105
}

\author{
J. DUGUNDJI \\ Department of Mathematics \\ University of Southern California \\ Los Angeles, California 90007 \\ RICHARD ARENS \\ University of California \\ Los Angeles, California 90024
}

\section{ASSOCIATE EDITORS}

E. F. BECKENBACH

B. H. NeUManN

F. WOLF

K. YosHIDA

\section{SUPPORTING INSTITUTIONS}

UNIVERSITY OF BRITISH COLUMBIA

CALIFORNIA INSTITUTE OF TECHNOLOGY

UNIVERSITY OF CALIFORNIA

MONTANA STATE UNIVERSITY

UNIVERSITY OF NEVADA

NEW MEXICO STATE UNIVERSITY

OREGON STATE UNIVERSITY

UNIVERSITY OF OREGON

OSAKA UNIVERSITY
UNIVERSITY OF SOUTHERN CALIFORNIA

STANFORD UNIVERSITY

UNIVERSITY OF TOKYO

UNIVERSITY OF UTAH

WASHINGTON STATE UNIVERSITY UNIVERSITY OF WASHINGTON

AMERICAN MATHEMATICAL SOCIETY NAVAL WEAPONS CENTER 


\section{Pacific Journal of Mathematics}

\section{Vol. 45, No. $1 \quad$ September, 1973}

William George Bade, Complementation problems for the Baire classes .......... 1

Ian Douglas Brown, Representation of finitely generated nilpotent groups ........ 13

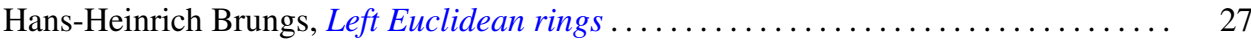

Victor P. Camillo and John Cozzens, A theorem on Noetherian hereditary rings ..... 35

James Cecil Cantrell, Codimension one embeddings of manifolds with locally flat

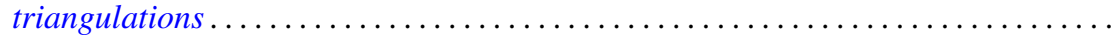

L. Carlitz, Enumeration of up-down permutations by number of rises . . . . . . . . . .

Thomas Ashland Chapman, Surgery and handle straightening in Hilbert cube

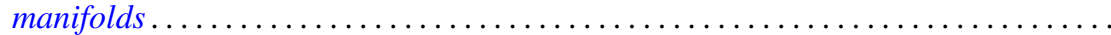

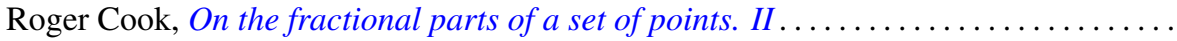

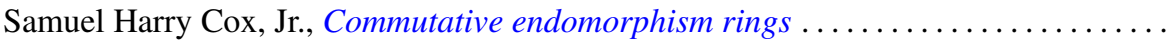

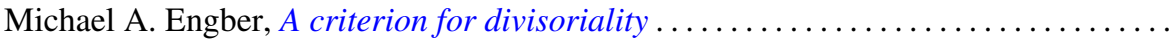

Carl Clifton Faith, When are proper cyclics injective . . . . . . . . . . . . . . 97

David Finkel, Local control and factorization of the focal subgroup . . . . . . . . . 113

Theodore William Gamelin and John Brady Garnett, Bounded approximation by

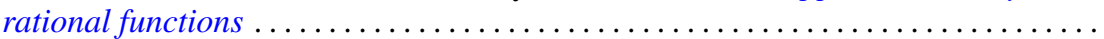

Kazimierz Goebel, On the minimal displacement of points under Lipschitzian

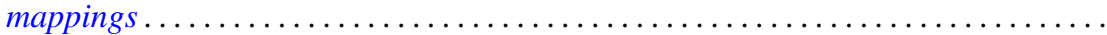

Frederick Paul Greenleaf and Martin Allen Moskowitz, Cyclic vectors for representations associated with positive definite measures: nonseparable

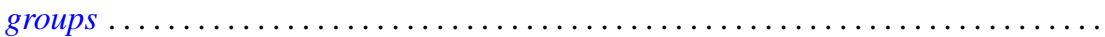

Thomas Guy Hallam and Nelson Onuchic, Asymptotic relations between perturbed linear systems of ordinary differential equations .

David Kent Harrison and Hoyt D. Warner, Infinite primes of fields and completions. .

James Michael Hornell, Divisorial complete intersections . ......

Jan W. Jaworowski, Equivariant extensions of maps ..............

John Jobe, Dendrites, dimension, and the inverse arc function .. .

Gerald William Johnson and David Lee Skoug, Feynman integrals of non-factorable

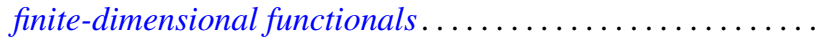

Dong S. Kim, A boundary for the algebras of bounded holomorphic functions ...... 269

Abel Klein, Renormalized products of the generalized free field and its derivatives ... 275

Joseph Michael Lambert, Simultaneous approximation and interpolation in $L_{1}$ and

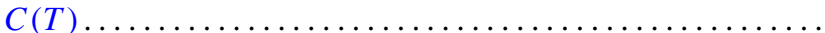

Kelly Denis McKennon, Multipliers of type $(p, p)$ and multipliers of the group

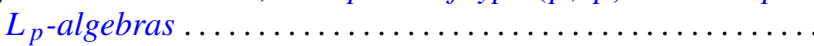

William Charles Nemitz and Thomas Paul Whaley, Varieties of implicative



Donald Steven Passman, Some isolated subsets of infinite solvable

Norma Mary Piacun and Li Pi Su, Wallman compactifications on E-completely

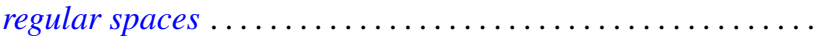

Jack Ray Porter and Charles I. Votaw, $S(\alpha)$ spaces and regular Hausdorff extensions....

Gary Sampson, Two-sided $L_{p}$ estimates of convolution transforms .

Ralph Edwin Showalter, Equations with operators forming a rig
Raymond Earl Smithson, Fixed points in partially ordered sets .

Victor Snaith and John James Ucci, Three remarks on symmetric products and

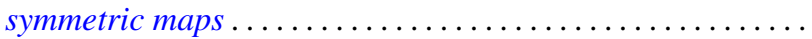

